\title{
Reverse Takotsubo cardiomyopathy in the setting of acute abdomen.
}

\author{
Legga A.E. MD, DESA 1, Manousakis S. MD 2 \\ ${ }^{1}$ Anaesthetist, County General Hospital of Agios Nikolaos, Dept of Anaesthesiology, Agios Nikolaos, Greece \\ ${ }^{2}$ Cardiologist, County General Hospital of Agios Nikolaos, Dept of Cardiology, Agios Nikolaos, Greece
}

\section{Background}

Reverse Takotsubo is a variant of the classic cardiomyopathy of transient left ventricular apical ballooning but typically presents at a younger age and is always associated with an emotional or physical stress as a trigger.

\section{Case report}

We report a case of a 32-year-old woman, with insignificant past medical history, presenting at the emergency department with acute pain at the abdomen and emesis. Preoperative evaluation included 12 lead electrocardiogram which revealed ST-depressions and T-wave inversions in precordial leads.Troponin T Hs, Cardiac Brain Natriuretic peptide and Creatinine Kinase-MB were elevated. The patient complained of severe pain at the abdomen with no dyspnea, chest pain or signs of heart failure.

Transthoracic echocardiogram revealed severe hypokinesis of the basal and mid segments of the heart and preserved contractility of the apex with an estimated ejection fraction of $35 \%$. There was also small pericardial effusion. The patient had had a transthoracic echocardiogram during her pregnancy, two months ago, which was normal.

Anaesthesia was uneventful using invasive monitoring of the arterial blood pressure, cardiac output and stroke volume variation monitoring to guide our fluids intraoperatively. Postoperatively the patient went to the coronary care unit. She had full cardiological recovery after fifteen days.
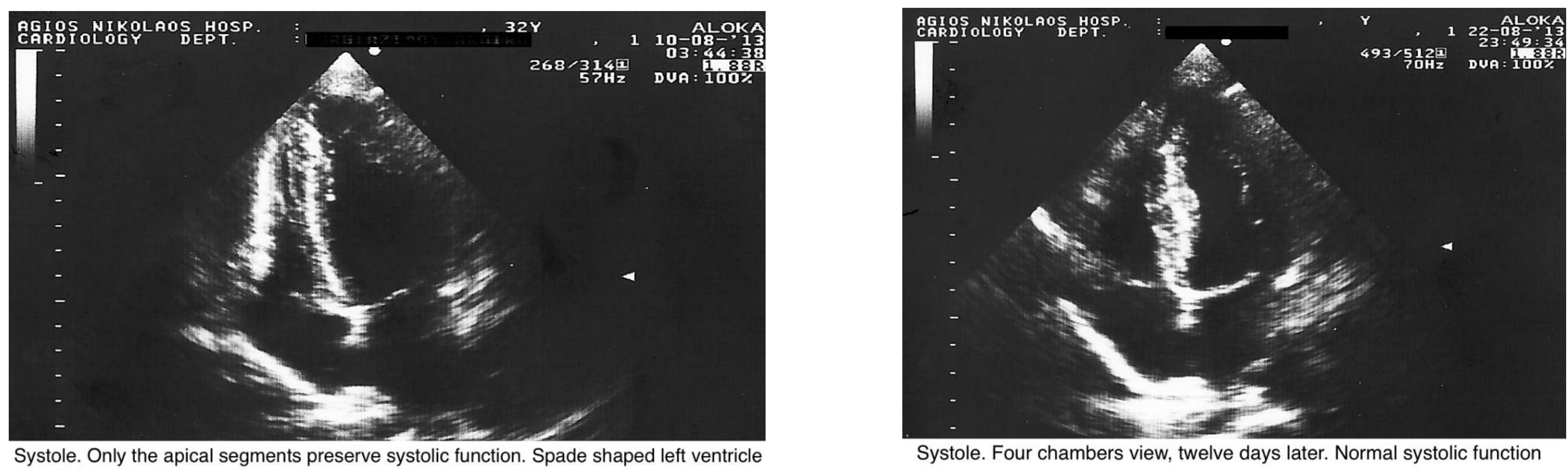

Systole. Four chambers view, twelve days later. Normal systolic function

\section{iscussion}

Emergency laparotomies are associated with high rates of postoperative complications and death with a 30 day mortality rate of $11 \%$. In an emergency situation it is easy to underestimate routine clinical findings such as changes in the ECG, especially when the patient is in pain,young, previously well and fit. Even P-Possum score would underestimate the individualised needs of our patient who needed specialised monitoring and cardiological post operative care. Takotsubo syndrome and its variants are referred more often in the literature and have a strong relation with excess of catecholamines and stress hormones.

\section{Conclusions}

There is a need for anaesthetists to be vigilant regarding Takotsubo syndrome because this warrants for different perioperative management. 